\title{
Cell-structure specific necrosis by optical-trap induced intracellular nuclear oscillation
}

\author{
X.X. Sun ${ }^{1}$, Z.L. Zhou ${ }^{1 *}$, C.H. Man $^{2}$, A.Y.H. Leung ${ }^{2}$ and A.H.W. Ngan ${ }^{1}$ \\ ${ }^{1}$ Department of Mechanical Engineering; \\ ${ }^{2}$ Li Ka Shing Faculty of Medicine; \\ University of Hong Kong, Pokfulam Road, Hong Kong, P.R. China \\ *Corresponding author (Email: zhouzhuolong@gmail.com)
}

\begin{abstract}
A drug-free procedure for killing malignant cells in a cell-type specific manner would represent a significant breakthrough for leukemia treatment. Here, we show that mechanically vibrating a cell in a specific oscillation condition can significantly promote necrosis. Specifically, oscillating the cell by a low-power laser trap at specific frequencies of a few $\mathrm{Hz}$ was found to result in increased death rate of $50 \%$ or above in different types of myelogenous leukemia cells, while normal leukocytes showed very little response to similar laser manipulations. The alteration of cell membrane permeability and cell volume, detected from ethidium bromide staining and measurement of intracellular sodium ion concentration, together with the observed membrane blebbing within 10 minutes, suggest cell necrosis. Mechanics modelling reveals severe distortion of the cytoskeleton cortex at frequencies in the same range for peaked cell death. The disruption of cell membrane leading to cell death is therefore due to the cortex distortion, and the frequency at which this becomes significant is celltype specific. Our findings lay down a new concept for treating leukemia based on vibration induced disruption of membrane in targeted malignant cells.
\end{abstract}

Keywords: optical trap; mechanical force; frequency; necrotic cell death; leukemia 


\section{Introduction}

Acute myeloid leukemia (AML) is one of the most lethal cancers worldwide, and untreated chronic myeloid leukemia (CML) can lead to the blast crisis phase and be fatal (Yong et al., 2006). Intensive chemotherapy of myeloid leukemia and allogeneic haematopoietic stem cell transplantation (HSCT) are the mainstays of treatment. However, this approach has reached an impasse and leukemia relapse is the commonest cause of treatment failure. Developing drug- or reagent-free physiotherapeutic strategies based on purely physical disturbances to kill leukemia cells may provide a novel therapeutic strategy for this disease with hitherto dismal outcome. A possible approach along this direction can be understood from the membrane-cytoskeletonnucleus structure shown in Figure $1(\mathrm{C}$ and $\mathrm{D})$. Due to the discreteness of the intracellular components, a vibrating disturbance at a specific frequency and amplitude may set certain components into a higher motion, thus resulting in excessive stresses which may lead to cell damage. In particular, large forces generated from nuclear oscillations in certain cell types with particular structures and components may be imparted on the cell membrane via the interconnecting cytoskeleton, leading to structural damages such as membrane blebbing (Majno and Joris, 1995), or possibly upsetting of the trans-membrane ion channels (Lang et al., 2007). Since the structures of different cell types are different, the response of different cell types to vibratory disturbances may also be different.

Based on the above hypothesis, in the present study we focus on the cell cytoskeleton and the surrounding membrane. We employed an optical trap (Ashkin et al., 1986) to purposely set the nucleus in different cell types (Gallagher et al., 1979; Lange et al., 1987; Lozzio and Lozzio, 1975; Tsuchiya et al., 1980; Wang et al., 1989) (see Table) into oscillations at different frequencies. This approach is remarkably different from previous attempts in which laser-beam scanning was blindly done over the cell without specifically trapping any cellular component such as the nucleus $(\mathrm{Ng}$ et al., 2013), or batches of cells in culture medium were vibrated via a motion generator (Zhou et al., 2016). Here, the massive nucleus is specifically trapped and oscillated by the optical tweezers, to investigate the effects of the cytoskeleton on the vibrationinduced cell death. Since in this work we do not specifically aim to examine the effects of the genetic difference between acute and chronic leukemia cells, chronic myeloid leukemia K562 cells, which are large leukemia cells with clearly observable subcellular 
structures, were chosen for in-depth analysis for the cell death mechanism under oscillations. Specifically, we applied a myosin II inhibitor Blebbistatin to modify the cytoskeletons of the K562 cells, prior to the laser manipulation experiments. Blebbistatin is known for its inhibiting effects on actin polymerization, kinetochore microtubule formation, mitotic spindle assembly (Kovacs et al., 2004; Limouze et al., 2004; Straight et al., 2003), and movement of actin filaments and microtubules leading to their unbundling (Burnette et al., 2008; Schaefer et al., 2008). In addition, the alteration of cell membrane permeability and cell volume were evaluated using staining by ethidium bromide (EB) and a $\mathrm{Na}^{+}$fluorescent dye Coro $\mathrm{Na}^{\mathrm{TM}}$ Green $\mathrm{AM}$ (Invitrogen) respectively, during the optical oscillations. Finally, the distortion of the cytoskeleton and the membrane cortex due to vibrational disturbances at frequencies ranging from 1 $\mathrm{Hz}$ to about $100 \mathrm{~Hz}$ is analyzed by finite-element modeling.

\section{Materials and methods}

\subsection{Cell preparation}

Myelogenous leukemia cell lines K562,OCI-AML-3, THP-1, HL60 and MV-4-11 (Lam et al., 2016), with a concentration of $\sim 10^{5}$ cells $/ \mathrm{ml}$ were cultured in a suspension manner in confocal dishes with fresh GIBCO RPMI 1640 media containing 10\% fetal bovine serum and $1 \%$ penicillin and streptomycin solution (Sigma-aldrich, St. Louis, USA) inside a $\mathrm{CO}_{2}$ incubator (Bionex, Model-VS-9160C, Seoul, South Korea), at a humidity of $95 \%$ and a temperature of $37^{\circ} \mathrm{C}$ for $\sim 24$ to 48 hours, and were then transferred into human blood plasma, pre-filtered by a $0.22 \mu \mathrm{m}$ filter, in confocal dishes. The cell passage numbers of all the leukemia cell lines used for the optical trap experiments are no more than 15, and those used for cell death determination and cytoskeleton staining are around 7 to 9 and 10 to 12, respectively. Normal leukocytes were isolated from healthy adult donors by a Ficoll ${ }^{\mathrm{TM}}$-Paque PLUS reagent assay (GE Healthcare Bio-Sciences, Piscataway, USA) and immediately used for optical oscillation. The leukocyte isolation procedure followed strictly the protocol accompanied with the Ficoll ${ }^{\mathrm{TM}}$-Paque PLUS reagent assay (Boyum, 1968). Approval for the leukocyte experiment was given by the Human Research Ethics Committee for Non-clinical Faculties of the University of Hong Kong (reference number EA240913). 


\subsection{Optical trap experiments}

Each confocal dish containing the suspending cells was placed inside a miniincubator (Okolab, 2GF-MIXER, Italy) installed onto the microscope stage of an optical tweezers system (Molecular Machines \& Industries, Eching, Germany). A schematic for the experiment is shown in Figure 1(A), and the mechanism of how a spherical object such as a nucleus is trapped is shown in Figure 1(B). Due to the Gaussian intensity profile of the beam, the particle experiences a lateral gradient force pulling it towards the beam center, and an axial scattering force pushing it in the direction of propagation of the beam towards the focal region (Dasgupta, 2012). The environment in the mini-incubator was also set to be the same as that for the Bionex $\mathrm{CO}_{2}$ incubator described above. The nuclei of individual cancer cells suspending in the culture dish were trapped by the laser beam of the Cell Manipulator (Nd:YAG laser, wavelength $1024 \mathrm{~nm}$, Eching, Germany) at a nominal trapping power of 0.56 watt. In the experiments, the cells were oscillated by the laser trap in suspension instead of when they are attached to a substrate. Laser-induced oscillation of the cell nucleus and membrane structure is in general not in phase and therefore a tethering force between the nucleus and membrane is produced. The beam was made to scan at frequencies of $1,4,8$ and $16 \mathrm{~Hz}$, with amplitudes of 4 and $8 \mu \mathrm{m}$. A nominal laser power rating of 0.56 watt was used because from a series of pre-tests using static-beam irradiation at different power ratings on K562 cells, this power rating was found to result in a low enough death rate without beam oscillations, which also served as the control group in the present study. Leukemia (K562, OCI-AML-3, THP-1, HL60 and MV-4-11) cells with spherical shape and diameters of $15 \pm 4 \mu \mathrm{m}$ were selected for the experiments to reduce the effects of cell size variation, and likewise, normal monocytes, granulocytes and macrophages of diameters $9 \pm 2,7 \pm 2$ and $12 \pm 2 \mu \mathrm{m}$, respectively were selected. All laser oscillations lasted for 10 minutes in the present study. A video showing typical laser driven oscillations of a cell at different frequencies is provided as Supplementary Material. Cell membrane blebbing observed under optical light during laser oscillation is considered as an indication of cell death (Weliky and Oster, 1990).

In the experiments, most of the cell nuclei were visible under optical light by adjusting the lens aperture to adapt to the plane where the nucleus can be seen (see Figure 2B). Besides, spherical objects under optical trap will tend to orientate automatically at the center of the laser trap as the axial gradient force produced by the 
laser trap pulls the particle towards the focal region (Neuman and Block, 2004). Since the cell nucleus is the biggest object inside the cell and nearly spherical, the nucleus will always be trapped at the end when the laser is shot on top of the center of a targeted suspended leukemia cell.

\subsection{Cell death determination}

\subsubsection{Ethidium bromide staining}

The cells were observed by a Nikon Eclipse Ti-U microscope (Tokyo, Japan) integrated into the optical tweezers system, at an objective lens magnification of $100 \times$. Every 2 minutes an optical micrograph was captured to detect any distortion in shape or membrane blebbing of the cells, as both phenomena may indicate cell death. To provide for more accurate determination of cell death, ethidium bromide (Sigma-aldrich, St. Louis, USA) staining also was used to indicate cell membrane integrity, since this reagent stains only the nucleus, but it cannot penetrate through the intact cell membrane of living cells (Liegler et al., 1995). Thus a cell becoming visible by fluorescence is a dying or dead cell with its membrane disintegrated. A final concentration of $50 \mu \mathrm{g} / \mathrm{ml}$ EB was added to the culture medium for cell staining, and fluorescence was detected by a Nikon Intensilight C-HGFI fluorescence exciter (Tokyo, Japan) integrated into the optical tweezers system under excitation and emission wavelengths of 320 and $600 \mathrm{~nm}$ respectively.

\subsection{2. $\mathrm{Na}^{+}$dye staining and cell volume evaluation}

To study the gain or loss of intracellular $\mathrm{Na}^{+}$concentration during the optical oscillations, a $\mathrm{Na}^{+}$fluorescent dye Coro $\mathrm{Na}^{\mathrm{TM}}$ Green $\mathrm{AM}$ (Thermo Fisher Scientific, Massachusetts, USA) was used to stain a batch of K562 cells at a final concentration of $5 \mu \mathrm{M}$. After mixing with the dye for 30 minutes, the cells were subjected to laser oscillations and fluorescence observation. The oscillation frequency and amplitude used were $4 \mathrm{~Hz}$ and $8 \mu \mathrm{m}$, respectively. Optical and fluorescent images were recorded every two minutes in the duration of the 10 minute oscillations. The excitation and emission wavelengths of Coro $\mathrm{Na}^{\mathrm{TM}}$ Green $\mathrm{AM}$ are $492 \mathrm{~nm}$ and $516 \mathrm{~nm}$ respectively. Cell volume regulation is also known to be an important process during cell death (Hoffmann et al., 2009). Therefore, the cell volumes of K562 cells were also measured 
based on the captured fluorescent micrographs. To minimize the effects of the initial cell size, only cells with diameter of around $17 \mu \mathrm{m}$ were used for this part of the work.

\subsection{Blebbistatin treatment and cytoskeleton staining}

To investigate the effect of cytoskeleton on the cell death, blebbistatin (Sigmaaldrich, St. Louis, USA) was added to K562 cells at a final concentration of $5 \mu \mathrm{M}$ four hours prior to the laser manipulation experiments. The treated cells were then subjected to the laser oscillations as described above, using oscillation frequencies of $4 \mathrm{~Hz}$ and amplitude of $8 \mu \mathrm{m}$.

The cytoskeleton comprising actin and tubulin filaments of K562 cells with and without the blebbistatin treatment was observed using a phalloidin-FITC conjugate solution (Sigma-aldrich, St. Louis, USA) (Cooper, 1987) and an anti- $\beta$-tubulin-Cy3 solution (Sigma-aldrich, St. Louis, USA). The phalloidin-FITC and anti- $\beta$-tubulin-Cy3 enable the visualization of F-actin and $\beta$-tubulin filaments under a fluorescence microscope, thus allowing the density of these filaments to be quantified. The confocal dishes cultured with suspending K562 cells were examined using a Zeiss laser-source fluorescence microscope integrated into a Bio-AFM microscope (JPK, NanoWizard II, Germany). The images were taken by at an objective lens magnification of $100 \times$. The emission wavelengths for FITC-phalloidin and anti- $\beta$-tubulin-Cy3 are $488 \mathrm{~nm}$ and 514 $\mathrm{nm}$ respectively. The fluorescent images of K562 cells with and without blebbistatin treatment were taken under the same exposure time.

\subsection{Statistical Analysis}

The death rates represent the number of dead cells divided by the total cells exposed to a certain frequency-amplitude condition. Cell death was judged from changes in cell morphology such as cell distortion or membrane blebbing observed under optical microscope. Two-tailed Chi-squared tests were performed for the death rates of different groups exposed to radiation with different frequencies and amplitudes. Two-population $t$-tests were performed on the nucleus translation results, the $\mathrm{Na}^{+}$dye staining and cell volume evaluation, and the cytoskeleton fluorescence intensity measurements. 


\section{Results}

Figure 1(A-D) show schematics of the present optical trap experiments. The cells studied were suspended in pre-filtered human blood plasma in confocal dishes. The optical beam size used was around $1 \mu \mathrm{m}$ and this is able to trap a $10 \mu \mathrm{m}$ diameter particle in a stable manner (Ashkin et al., 1986). The present leukemia cells were larger than ten microns in general (Baddour and Kolios, 2007; Fleck et al., 2005; Gabriele et al., 2009; Zhou et al., 2014b; Zhou et al., 2012), and for the present experiments, cells of a narrower size range of $15 \pm 4 \mu \mathrm{m}$ and of spherical shape were chosen for the leukemia species, in order to ensure uniformity. In the laser manipulation experiments, a 0.56 watt laser power was employed, at which the cell death rate was not significantly higher than normal upon irradiation by a non-vibrating beam for 10 minutes, which was the duration in all the laser vibration experiments.

Figure 2(A) shows the measured death proportion of the five leukemia cell types studied (K562, OCI-AML-3, THP-1, HL60, MV-4-11), as well as that for normal monocytes, granulocytes and macrophages as control, all oscillated at beam amplitude of 4 or $8 \mu \mathrm{m}$, and frequencies of 0 (i.e. no scanning), 1, 4, 8, 16 and $24 \mathrm{~Hz}$. For the leukemia cells, their death rate under laser oscillations all exhibits significant difference from the control of static laser radiation (i.e. data at $0 \mathrm{~Hz}$ ) according to two-tailed Chisquare tests, and the death rate peaks at $4 \mathrm{~Hz}$ for K562, $8 \mathrm{~Hz}$ for THP-1, HL60 and MV4-11, and $16 \mathrm{~Hz}$ for OCI-AML-3. Interestingly, in Figure 2(A), normal monocytes, granulocytes and macrophages do not show significant differences in death rate under oscillations over the same frequency range, compared to static laser irradiation. This may indicate a significant structural difference between the less differentiated and diseased leukemia cells and the well differentiated normal leukocytes. In any case, the results here show that the oscillation-induced cell death is frequency and cell-type dependent. The optical micrographs in Figure 2(B) shows the death process of typical cells in different species under laser oscillations of $8 \mu \mathrm{m}$ amplitude and at frequencies corresponding to their maximum death rate in Figure 2(A). It can be clearly seen that membrane blebbing and cell distortion occurred in the leukemia cell types after 6 to 8 minutes of laser oscillations, but not in the normal leukocytes during the 10-minute manipulation by laser. A video showing the typical membrane blebbing in a K562 cell during oscillations at $4 \mathrm{~Hz}$ and $8 \mu \mathrm{m}$ amplitude input is available as Supplementary Material. 
Figure 3(A) shows the optical and fluorescent micrographs of a typical K562 cell undergoing death during the 10 minute oscillations with a further 5 minute observation time without the laser irradiation. The cell started to distort after 6 minutes of laser oscillations, and the nucleus became eminent with the EB staining after 8 minutes of laser oscillations. Obvious increment of the cell volume after the oscillations can be observed by comparing the optical micrographs at 15 minutes and 0 minute, while in the control group with static radiation only, no EB fluorescence was detected. To further study the alteration of membrane permeability and cell volume, Coro $\mathrm{Na}^{\mathrm{TM}}$ Green $\mathrm{AM}$ dye for $\mathrm{Na}^{+}$ions was applied to a batch of K562 cells. The measured ion intensities inside three K562 cells with and without laser oscillations at frequency of $4 \mathrm{~Hz}$ and amplitude of $8 \mu \mathrm{m}$ are compared in Figure 3(B,C). Figure 3(B) shows the typical micrographs indicating the $\mathrm{Na}^{+}$intensities, which are quantified in Figure 3(C). It can be seen that the intracellular $\mathrm{Na}^{+}$intensity of the $\mathrm{K} 562$ cell exposed to static radiation exhibited a very mild rise with time, which may be due to the continued staining effects of the dye or its continuous uptake by the cell. On the other hand, the cell exposed to oscillatory radiation yet with its membrane still intact exhibited a much faster increase of the intracellular $\mathrm{Na}^{+}$intensity over the irradiation time, indicating that $\mathrm{Na}^{+}$ continuously flowed into the cell during the laser oscillations. A third K562 cell studied had its membrane disintegrating after 4 minutes of laser oscillations, and it can be seen from Figure $3(\mathrm{~B}, \mathrm{C})$ that the measured intracellular $\mathrm{Na}^{+}$intensity underwent a significant drop after membrane disintegration began. Since the extracellular $\mathrm{Na}^{+}$ concentration should be higher than the intracellular, the observed drop in the dyed $\mathrm{Na}^{+}$ intensity after membrane disintegration is likely to be due to the loss of the visible hydrolyzed $\mathrm{Na}^{+}$Coro $\mathrm{Na}^{\mathrm{TM}}$ Green, the precursor of which, namely, the Coro $\mathrm{Na}^{\mathrm{TM}}$ Green AM dye, experienced a hydrolysis reaction triggered by the intracellular esterases and lost the acetoxymethyl (AM) group (Amorino and Fox, 1995). The results in Figure 3(B,C) nevertheless suggest that the laser oscillations can disrupt the cell membrane, such as upsetting the $\mathrm{Na}^{+}$related transmembrane ion channels of the stretch activated or tethered types (Sackin, 1995; Zanini and Gopfert, 2013). The cell size increment was measured by the Image $1.46 \mathrm{r}$ software from the $\mathrm{Na}^{+}$fluorescent images captured during the experiments. As shown in Figure 3(D), a rapid increase in the cell size was observed in the cell subjected to laser oscillations yet with its membrane intact, indicating continuous water uptake during the laser oscillation process. This proves that the intracellular osmotic pressure was increased by the laser oscillations, which agrees 
well with the increase in intracellular $\mathrm{Na}+$ in Figure 3(B,C). Again, during the laser oscillation process, the cell membrane was disrupted, and/or the stretch activated or tethered transmembrane ion channels may be forced open, causing $\mathrm{Na}^{+}$influx due to the higher extracellular $\mathrm{Na}^{+}$concentration, and water influx as well due to the increased intracellular osmotic pressure. Cell swelling, or cell volume increase CVI, is a sign for necrosis (Barros et al., 2001; Barros et al., 2003). The findings here also indicate strong evidence for necrotic cell death, since membrane integrity would be maintained in early apoptosis (Patel et al., 2006).

Figure 4(A) shows a pair of optical micrographs taken before and after one half of a typical cycle of laser scanning on a K562 cell, where a relative displacement between the nucleus and membrane can be noted as indicated by the difference in lengths of the line markers shown in the micrographs. Figure 4(B) shows statistically that the nucleus translation existed after the K562 cells was subjected to the laser oscillation $(p<0.05)$. The nucleus translation caused by laser-trap induced oscillation shown in Figure 4(B) implies that a stretching motion driven by the optical trap occurs on the membrane-cell cytoskeleton-nucleus system, and this should result in forces transferred to the cell membrane that may lead to cell damage, since the nucleus is tightly connected with the cytoskeleton network, rather than an object isolated from the cell cytoskeleton and other subcellular components.

The death proportion of K562 cells treated with blebbistatin and oscillated at laser beam amplitude of $8 \mu \mathrm{m}$ was also measured as shown in Figure 2(A). In comparison with K562 cells without blebbistatin treatment, the cells treated with blebbistatin no longer show a peak in death proportion at $4 \mathrm{~Hz}$, and their death proportion under laser oscillations actually remains close to that of the control under static laser irradiation. Figure 5(A) shows the F-actin and $\beta$-tubulin filaments of K562 cells stained by phalloidin-FITC and anti- $\beta$-tubulin-Cy3 under the fluorescent microscope, with and without the blebbistatin treatment. Figure 5(B) shows that the fluorescence intensities of F-actin filaments and microtubules of the blebbistatin treated group are significantly lower than those of the control group without blebbistatin treatment $(\mathrm{p}<0.001)$, thus indicating significant weakening of the cytoskeleton structure by the blebbistatin drug treatment (Burnette et al., 2008; Kovacs et al., 2004; Limouze et al., 2004; Schaefer et al., 2008; Straight et al., 2003). The blebbistatin treatment was found to significantly reduce the cell stiffness. According to the model in Figure 1(C), a reduced density of 
F-actin and tubulin filaments should result in a reduction of the forces transmitted from the oscillating nucleus to the cell membrane, thus reducing the stretching of the latter which may explain the reduced death rate of the blebbistatin treated K562 cells in Figure 2(A). The results in Figures 2(A) and 4 collectively indicate that cells with a weakened cytoskeleton may survive better under oscillations.

\section{Discussion}

In the present experiments, the laser beam was shot directly onto the nucleus and scanned in a low frequency range, setting the latter into vibrations, albeit with an amplitude different from that of the beam, due to the fact that the laser trap behaves as a harmonic spring (Andersson et al., 2007), so that the motion of the trapped nucleus is the dynamic response of such a harmonic force, the inertia of the nucleus and the damping and stiffness of the intracellular medium in which it moves. The resultant optimal frequency for peak cell death was then found to be only a few $\mathrm{Hz}$ (Figure 2(A)). As stated above, the laser power used in the present work was too low to kill the trapped cell directly by its irradiation effect. Although the laser irradiation may increase the membrane tension or reduce its flexibility (Batchelder et al., 2011), this factor cannot explain the elevated death rate under oscillatory laser scanning, compared with static irradiation by the same laser power for the same duration (Figure 2(A)). Also, cell death occurred after short laser manipulation for 10 minutes or sooner, and during such short times, genetic responses such as gene expression and signal transduction could not have played a significant role (Chien et al., 1998). The only plausible explanation for the cell death would be purely mechanically induced structural damages in certain cellular components, due to the large mechanical stresses set up as a result of the motion of the nucleus. Enhanced cell death triggered by vibrating magnetic particles externally delivered to the cells has been reported (Kim et al., 2010; Wang et al., 2013). However, the present study demonstrates that the oscillations of the entire cell structure within the cell volume can lead to enhanced cell death - in this method, no foreign reagent needs to be involved.

Figure 3(B,C) indicate that laser oscillations disrupt the cell membrane causing uptake of $\mathrm{Na}^{+}$ions into the cell, and Figure 3(D) suggests that water uptake also increased, possibly as a result of the increased osmotic pressure accompanying the increased intracellular $\mathrm{Na}^{+}$content. Therefore, the present results collectively suggest 
that the excessive oscillations of the cell nucleus at designated driving frequencies in the $\mathrm{Hz}$ range impart large mechanical stresses on the cell membrane via the cytoskeleton and cytoplasm as illustrated in Figure 1(C and D), disrupting or even rupturing the cell membrane. In particular, stretch-activated/tethering ion channels have been suggested to play an important role in stress related cell death (Sachs, 2010). The oscillations may also lead to local pressure unbalances between the cytoplasm and the extracellular medium, leading to cell membrane blebbing and eventual cell death (Weliky and Oster, 1990). The cytoskeleton results in Figure 5 provide further evidence for this cell death mechanism under vibrations. As the cytoskeleton is tightly linked to the nucleus through the actin-membrane proteins on the cell membrane (Rimm et al., 1995), a weakened cytoskeleton with reduced filament density should be less effective in transmitting stresses to the cell membrane from the oscillating nucleus. The results in Figure 5 show that reducing the amount of F-actin filaments by using blebbistatin to inhibit actin polymerization indeed led to a decreased death proportion of K562 cells under oscillations. The different death rates of the leukemia cells and normal leukocytes measured from the laser oscillation experiment suggest structural differences between the diseased and normal cells. Leukemic myeloblasts are characterized by high nuclearcytoplasm (N/C) ratio, open chromatin and prominent nucleoli and morphologically distinct from granulocytes or monocytes (Alberts et al., 2002). Erythrocytes could not be killed (data not shown) in all the tested frequencies in Figure 2(A), probably because they do not have nuclei. In addition, the cytoskeletons are also obviously different among the different tested cell types according to the different elastic moduli measured (Zhou et al., 2014b). Blebbistatin treatment reduced the actin and the tubulin filaments in the cytoskeleton of K562 cells (shown in Figure 5), resulting in reduction of both the cell stiffness and force delivery from nucleus to membrane - in other words, a cell with reduced cytoskeleton stiffness would have reduced forces transmitted to the membrane from the oscillating nucleus, thus the damage of the membrane would be smaller. This again explains why Erythrocytes could not be killed by laser oscillation. These factors are likely to significantly affect the vibration characteristics of the nucleus or other intracellular components according to the nucleus-cytoskeleton-membrane model suggested above in Figure 1(C and D).

To understand how exactly oscillations of the laser trap can disrupt the nucleuscytoskeleton-membrane system, modeling work was conducted. In the present experiments, the laser beam was made to incident on and vibrate the nucleus, and hence 
the motion of the nucleus induced by the optical trap needs to be understood first. The nucleus is forced to vibrate within the intracellular medium which is a complex viscoelastic medium, both the elastic and loss modulus of which are frequency dependent (Guo et al., 2013) according to a power-law relation (Balland et al., 2006). In addition, for small oscillation amplitudes, the optical trap behaves as a linear spring between the laser beam and the nucleus (McAlinden et al., 2014). As the nucleus is set to oscillate in the horizontal direction, its motion $x(t)$ can be estimated by a onedimensional model of forced vibration with damping, and its oscillation amplitude $x_{o}(\omega)$ can be solved analytically as

$$
x_{o}(\omega)=\frac{k_{\text {trap }} A}{\sqrt{\left(k+k_{\text {trap }}-M \omega^{2}\right)^{2}+(\beta \omega)^{2}}}
$$

where $k_{\text {trap }}$ is the optical trap stiffness of $\sim 33.6 \mathrm{pN} / \mu \mathrm{m}$ (see Supplementary Materials (I) for details), $A=8 \mu \mathrm{m}$ is the vibration amplitude of the optical trap, and $M$ is the mass of the nucleus. $k$ and $\beta$ are respectively the stiffness and damping coefficient of the intracellular medium and they can be obtained from the elastic and loss moduli of the intracellular medium as $k=156.56 \omega^{0.3} \times 10^{-6} \mathrm{~N} / \mathrm{m}$ and $\beta=31.312 \omega^{-0.7} \times$ $10^{-6} \mathrm{Ns} / \mathrm{m}$, i.e. they are both frequency dependent (see Supplementary Material (I)). As a result, the oscillation amplitude of the nucleus decreases monotonously as the frequency increases, as shown by the black curve in Figure 6(A).

The cell membrane is composed of a phospholipid bilayer supported by the cytoskeleton cortex, and the latter evidently determines the spherical shape of the leukemia cell types used in the present experiments. As the bending stiffness of the lipid bilayer, which is $\sim 10^{-20} \mathrm{~J}$ (Rawicz et al., 2000), is orders of magnitude lower than that of the underlying cortex which is $K=\frac{E t^{3}}{12\left(1-v^{2}\right)} \sim 7 \times 10^{-17} \mathrm{Nm}$, where $t \sim 0.2 \mu \mathrm{m}$ is the thickness of the cell membrane, $v$ is Poisson ratio $(\sim 1 / 3)$ and $E=74.62 \mathrm{~Pa}$ is the elastic modulus measured by optical tweezers indentation experiments (Zhou et al., 2014a), the response of the more rigid cortex to the vibratory disturbances becomes the key to understand the membrane distortion during cell oscillations. A finite-element model of the cortex was constructed and put into explicit dynamic simulation, the details of which are described in the Supplementary Material (II). Vibratory disturbances of various frequencies and frequency dependent amplitudes $x_{o}(\omega)$ calculated from eqn. (1) were applied to the cortex model. Drag forces representing the viscosity of surrounding culture medium were also exerted on the elements composing 
the cortex. The simulation results indicate that when the frequency is very low at about $1 \mathrm{~Hz}$, the cortex can almost maintain its original spherical shape well during its vibratory motion in the same direction of the applied disturbance. However, when the frequency increases to the 4 to $8 \mathrm{~Hz}$ range, more serious distortions happen to the cortex. If the oscillating frequency is continuously increased to $32 \mathrm{~Hz}$, the distortion of the cortex becomes not as significant as that for 4 and $8 \mathrm{~Hz}$. To quantify the distortion phenomenon, the root-mean-square values of the nodal displacements at 0.5 cycle are calculated, when the distortion is the most serious. The cortex is actuated by a displacement $x_{o} \sin (\omega t)$ through the actuation nodes, but due primarily to the drag force, the nodes on the cortex do not move in phase with the actuation nodes, and the root-mean-square of the nodal displacements can reflect the stretching between the actuation nodes and the driven nodes on the cortex. The results are plotted in Figure 6(A). It can be seen that although the amplitude of the nucleus decreases monotonously with increasing frequency, cortex distortion exhibits a peak at around the 4 to $8 \mathrm{~Hz}$ range. This result is consistent with the cortex distortions and the peak death rate observed in the experiments. Therefore, according to the simulation results, for a specific cortex, there exists a specific frequency at which the distortion of the cortex is more serious than that at other frequencies.

Although a peak response at a specific frequency is usually related to resonance, it is important to note that the situation depicted in Figure 6(A) is not an intrinsic resonance behavior of the cortex model in our simulations. By oscillating the cortex model using the same actuating nodes but with the viscosity turned off, structural resonance was found to occur at about $60 \sim 80 \mathrm{~Hz}$, which is significantly higher than the 4-8 Hz frequency for peak distortion in Figure 6(A). Previously, we have shown that by using optical tweezers to blindly scan K562 cells without specifically trapping their nuclei, high cell death rates occurred at specific frequencies of a hundred $\mathrm{Hz}$ or above (Ng et al., 2013; Zhou et al., 2016). In that study, the frequencies used were too discrete to reveal any peak death behavior in the range of a few $\mathrm{Hz}$ as observed in the present work, and also, the nuclei were not purposely trapped, but nevertheless, cell destruction in the range of a hundred $\mathrm{Hz}$ or so was demonstrated. Recently, we also showed that shaking K562 and other suspended cell types in batches in culture medium by a vibrator (hence no longer single-cell manipulation) at $100 \mathrm{~Hz}$ would lead to enhanced transfection of nano-materials through disruption of the cell membrane (Zhou et al., 2016). These two previous studies (Ng et al., 2013; Zhou et al., 2016) therefore indicate 
the existence of other sensitive frequencies in the range of a hundred $\mathrm{Hz}$ or so, which is close to the resonance frequency of the cortex model in the present simulation.

Whereas the peak cortex disruption (the gray curve) in Figure 6(A) is not an intrinsic resonance behavior of the cortex, the blue curve shows that, as a result of the viscoelasticity of the intracellular medium, the oscillation amplitude of the driven nucleus decreases monotonically with frequency, and so does the actuation amplitude of the cortex deformation (see eqn. (1) where $k$ and $\beta$ depends on frequency $\omega$ ). The peak in the cortex distortion is therefore a consequence of the fact that the actuation amplitude is not constant but is monotonically decreasing with frequency. Thus, at small frequencies, although the actuation amplitude is high the cell moves as if it is a rigid body without much distortion of the cortex (see simulation movie at $2 \mathrm{~Hz}$ as Supplementary Material). On the other hand, at high frequencies, the actuation amplitude is small, so the cortex distortion is also small (see simulation movies at 32 and $128 \mathrm{~Hz}$ as Supplementary Materials). Therefore, at some intermediate frequency of a few $\mathrm{Hz}$ (see simulation movie at $8 \mathrm{~Hz}$ ), the cortex distortion is maximum. Therefore, to sum up, the peak cortex distortion at $8 \mathrm{~Hz}$ or so in the present work is not an intrinsic resonance response of the cortex, but is a consequence of the fact that the laser trap drives the nucleus to move with an amplitude that is monotonically decreasing with frequency, as a result of the viscoelasticity of the intracellular medium. In any case, the experimental results and the analysis above still show that trapping the nucleus and oscillating it at a few $\mathrm{Hz}$ can promote cell death due to cortex distortions. Significant deformation of the cortex should induce significant disruptions of the soft phospholipid bilayer supported by the cytoskeleton cortex, and upsetting of the cell membrane may lead to necrosis due to excessive ion and water uptake.

The results shown in Figure 6(A) are from a specific finite-element model constructed for a given cortex structure, and it is evident that such a cortex structure well represents the leukemia cells studied in Figure 2(A), since the same range of peak frequencies are predicted. However, Figure 2(A) also indicates that different cell types exhibit different specific frequencies for peak cell death. To explain this, we note that the distortion of the cortex can be represented by the relative motion between any two nodes on it, say, an actuation node and a typical driven node nearby. While the displacement of an actuation node is $x_{o} \sin (\omega t)$ where $x_{o}$ is given by eqn. (1) and $\omega$ is the angular frequency, the displacement of a typical driven node under damping effect 
is $z(t)=Z \sin (\omega t+\varphi)$, where $Z=\frac{x_{o}}{\sqrt{\left(1-r^{2}\right)^{2}+(2 \zeta r)^{2}}}$ is the amplitude, $\varphi=\tan ^{-1} \frac{2 c r}{1-r^{2}}$ is the phase lag, $r=\omega / \omega_{n}$ with $\omega_{n}$ being the natural frequency of the cortex structure, $\zeta=\frac{c}{2 m \omega_{n}}$ is the damping ratio, $m$ is the mass of the cortex, $c=2 \pi R \eta$ is the damping coefficient due to the culture medium, $\eta$ is the viscosity coefficient of the culture medium, and $R$ is the radius of the cortex (for details of the parameters, see Supplementary Material (I, II)). The relative motion between an actuation node and a driven node is therefore

$$
x_{o} \sin (\omega t)-Z \sin (\omega t+\varphi)=x_{o} \sin (\omega t)-\frac{x_{o}}{\sqrt{\left(1-r^{2}\right)^{2}+(2 \zeta r)^{2}}} \sin (\omega t+\varphi)
$$

Eqns. (1) and (2) then indicate that, apart from the stiffness and vibration amplitude of the optical trap, the peak frequency should be a cell-type or even cell-cycle dependent quantity, since it is related to several intrinsic properties of a specific cell, such as mass, stiffness and damping coefficient of the intracellular medium, and the natural frequency which is related to the radius, elastic modulus, and thickness of the cortex (Soedel, 2004). As an illustration, Figure 6(B) shows plots of the relative motion in eqn. (2) at $\omega t=\frac{\pi}{2}$ when the distortion is most serious, calculated using the model parameters given in Supplementary Materials (I, II) at four different values of the natural frequency (NF) of the cortex structure. It can be seen that a cytoskeleton cortex with a smaller natural frequency exhibits higher deformation upon forced oscillations, and vice versa. As the natural frequency of a spherical shell is proportional to $\frac{1}{R} \sqrt{\frac{E}{\rho}}$ (Soedel, 2004), the natural frequency of the cortex of a leukemia cell can be expected to be smaller than that of a normal cell, since the mass density $\rho$ and radius $R$ should be higher. Leukemia cells are thus expected to undergo larger cortex deformation upon vibrations than normal cells, and hence their higher death rates upon oscillations as experimentally observed in Figure 2(A) can be explained. It should be noted, however, that only the oscillation and natural frequencies of the cytoskeleton cortex are considered in Figure 6(B), and in order to analyze different cell types in general, their mass as well as other parameters will have to be known precisely. In any case, the present results and analysis suggest the possibility of using mechanical vibrations of specific frequencies to trigger 
the necrosis of targeted cell types, although more data from different cell types need to be gathered in order to realize such a technique in clinical practice.

Finally, the laser trap experiments described thus far were designed to study the fate of individual cells under forced oscillations in a well-defined manner, but the single-cell manipulation involved would be too inefficient if the purpose is to kill malignant cells in large quantities. As an illustration on how the latter aim may be achieved, we also attempted employing a simple sound-transducer setup to transmit sonic vibrations to batches of K562 and HL60 leukemia cells and normal leukocytes, cultured in a liquid medium with a viscosity similar to that of human blood plasma. Details are given in the Supplementary Material (III), and the results show that batches of these cells can be killed by sonic vibrations, and responses of different cell types are different. More research is needed to determine the specific responses of different cell types to mechanical vibrations of different nature and frequencies, but the present work has laid down a scientific basis for possibility of developing vibration-based physiotherapeutic strategies for treating leukemia. This study proposes an approach in which suspended myeloid leukemia cells can be killed at specific vibrational conditions according to their cell structure difference. For translation into clinic adaptation, an device may be developed for renewing bloods from patients by the present oscillation treatment which preferentially kills specific types of myeloid leukemia cells at specific intensity, amplitude and frequency conditions.

\section{Conclusions}

Optical oscillations of the nuclear parts of leukemia cells at low frequencies of a few $\mathrm{Hz}$ can trigger necrosis. The cell death induced by optical oscillations is frequency, amplitude and cell type dependent - all leukemia cell types studied exhibit a high death rate at certain frequencies of a few $\mathrm{Hz}$, whereas normal leukocytes are much less affected by laser oscillations. The present results suggest that death of leukemia cells is induced by nuclear oscillations which transmit stresses to upset the cell membrane, leading to ion influx and excessive water uptake by osmosis.

\section{Author contributions}

Z.L. Zhou designed and performed the experiments, analyzed the data and drafted part of the manuscript; X.X. Sun designed and performed the simulation work and drafted 
part of the manuscript; A.H.W. Ngan coordinated the project and discussion, provided laboratory support, and finalized the manuscript; C.H. Man gave valuable suggestions and discussion for the experiment; A.Y.H. Leung provided the leukemia cells, gave valuable suggestions, discussion and laboratory support for the experiments. All authors reviewed the manuscript.

\section{Acknowledgments}

The work described in this paper was supported by an endowment fund from the Kingboard Professorship in Materials Engineering for A.H.W. Ngan.

\section{REFERENCES}

Alberts, B., Johnson, A., Lewis, J., Raff, M., Roberts, K., Walter, P., 2002. "Leukocyte functions and percentage breakdown" Molecular Biology of the Cell (4th ed.). Garland Science, New York.

Amorino, G.P., Fox, M.H., 1995. Intracellular Na+ Measurements Using Sodium Green Tetraacetate with Flow-Cytometry. Cytometry 21, 248-256.

Andersson, M., Madgavkar, A., Stjerndahl, M., Wu, Y.R., Tan, W.H., Duran, R., Niehren, S., Mustafa, K., Arvidson, K., Wennerberg, A., 2007. Using optical tweezers for measuring the interaction forces between human bone cells and implant surfaces: System design and force calibration. Rev Sci Instrum 78.

Ashkin, A., Dziedzic, J.M., Bjorkholm, J.E., Chu, S., 1986. Observation of a singlebeam gradient force optical trap for dielectric particles. Opt Lett 11, 288.

Baddour, R.E., Kolios, M.C., 2007. The fluid and elastic nature of nucleated cells: implications from the cellular backscatter response. J Acoust Soc Am 121, EL16-22.

Balland, M., Desprat, N., Icard, D., Féréol, S., Asnacios, A., Browaeys, J., Hénon, S., Gallet, F., 2006. Power laws in microrheology experiments on living cells: Comparative analysis and modeling. Physical Review E 74, 021911.

Barros, L.F., Hermosilla, T., Castro, J., 2001. Necrotic volume increase and the early physiology of necrosis. Comparative biochemistry and physiology. Part A, Molecular \& integrative physiology 130, 401-409.

Barros, L.F., Kanaseki, T., Sabirov, R., Morishima, S., Castro, J., Bittner, C.X., Maeno, E., Ando-Akatsuka, Y., Okada, Y., 2003. Apoptotic and necrotic blebs in epithelial cells display similar neck diameters but different kinase dependency. Cell Death Differ 10, 687-697.

Batchelder, E.L., Hollopeter, G., Campillo, C., Mezanges, X., Jorgensen, E.M., Nassoy, P., Sens, P., Plastino, J., 2011. Membrane tension regulates motility by controlling lamellipodium organization. Proc Natl Acad Sci U S A 108, 11429-11434.

Boyum, A., 1968. Isolation of Leucocytes from Human Blood - a 2-Phase System for Removal of Red Cells with Methylcellulose as Erythrocyte-Aggregating Agent. Scand J Clin Lab Inv S 21, 9-\&. 
Burnette, D.T., Ji, L., Schaefer, A.W., Medeiros, N.A., Danuser, G., Forscher, P., 2008. Myosin II activity facilitates microtubule bundling in the neuronal growth cone neck. Developmental cell 15, 163-169.

Chien, S., Li, S., Shyy, Y.J., 1998. Effects of mechanical forces on signal transduction and gene expression in endothelial cells. Hypertension 31, 162-169.

Cooper, J.A., 1987. Effects of cytochalasin and phalloidin on actin. J Cell Biol 105, 1473-1478.

Dasgupta, R., 2012. Optical tweezers: light for manipulating microscopic world. J Phys Conf Ser 365.

Fleck, R.A., Romero-Steiner, S., Nahm, M.H., 2005. Use of HL-60 cell line to measure opsonic capacity of pneumococcal antibodies. Clinical and diagnostic laboratory immunology 12, 19-27.

Gabriele, S., Benoliel, A.M., Bongrand, P., Theodoly, O., 2009. Microfluidic investigation reveals distinct roles for actin cytoskeleton and myosin II activity in capillary leukocyte trafficking. Biophys J 96, 4308-4318.

Gallagher, R., Collins, S., Trujillo, J., McCredie, K., Ahearn, M., Tsai, S., Metzgar, R., Aulakh, G., Ting, R., Ruscetti, F., Gallo, R., 1979. Characterization of the continuous, differentiating myeloid cell line (HL-60) from a patient with acute promyelocytic leukemia. Blood 54, 713-733.

Guo, M., Ehrlicher, A.J., Mahammad, S., Fabich, H., Jensen, M.H., Moore, J.R., Fredberg, J.J., Goldman, R.D., Weitz, D.A., 2013. The role of vimentin intermediate filaments in cortical and cytoplasmic mechanics. Biophysical journal 105, 1562-1568. Hoffmann, E.K., Lambert, I.H., Pedersen, S.F., 2009. Physiology of cell volume regulation in vertebrates. Physiol Rev 89, 193-277.

Kim, D.H., Rozhkova, E.A., Ulasov, I.V., Bader, S.D., Rajh, T., Lesniak, M.S., Novosad, V., 2010. Biofunctionalized magnetic-vortex microdiscs for targeted cancercell destruction. Nature materials 9, 165-171.

Kovacs, M., Toth, J., Hetenyi, C., Malnasi-Csizmadia, A., Sellers, J.R., 2004. Mechanism of blebbistatin inhibition of myosin II. J Biol Chem 279, 35557-35563.

Lam, S.S., Ho, E.S., He, B.L., Wong, W.W., Cher, C.Y., Ng, N.K., Man, C.H., Gill, H., Cheung, A.M., Ip, H.W., So, C.C., Tamburini, J., So, C.W., Ho, D.N., Au, C.H., Chan, T.L., Ma, E.S., Liang, R., Kwong, Y.L., Leung, A.Y., 2016. Homoharringtonine (omacetaxine mepesuccinate) as an adjunct for FLT3-ITD acute myeloid leukemia. Sci Transl Med 8, 359ra129.

Lang, F., Foller, M., Lang, K., Lang, P., Ritter, M., Vereninov, A., Szabo, I., Huber, S.M., Gulbins, E., 2007. Cell volume regulatory ion channels in cell proliferation and cell death. Methods in enzymology 428, 209-225.

Lange, B., Valtieri, M., Santoli, D., Caracciolo, D., Mavilio, F., Gemperlein, I., Griffin, C., Emanuel, B., Finan, J., Nowell, P., et al., 1987. Growth factor requirements of childhood acute leukemia: establishment of GM-CSF-dependent cell lines. Blood 70, 192-199.

Liegler, T.J., Hyun, W., Yen, T.S., Stites, D.P., 1995. Detection and quantification of live, apoptotic, and necrotic human peripheral lymphocytes by single-laser flow cytometry. Clin Diagn Lab Immunol 2, 369-376.

Limouze, J., Straight, A.F., Mitchison, T., Sellers, J.R., 2004. Specificity of blebbistatin, an inhibitor of myosin II. Journal of muscle research and cell motility 25, 337-341.

Lozzio, C.B., Lozzio, B.B., 1975. Human chronic myelogenous leukemia cell-line with positive Philadelphia chromosome. Blood 45, 321-334.

Majno, G., Joris, I., 1995. Apoptosis, oncosis, and necrosis. An overview of cell death. Am J Pathol 146, 3-15. 
McAlinden, N., Glass, D.G., Millington, O.R., Wright, A.J., 2014. Accurate position tracking of optically trapped live cells. Biomedical optics express 5, 1026-1037.

Neuman, K.C., Block, S.M., 2004. Optical trapping. Rev Sci Instrum 75, 2787-2809.

Ng, K., Zhou, Z., Ngan, A., 2013. Frequency-dependent cell death by optical tweezers manipulation. Journal of cellular physiology 228, 2037-2041.

Patel, V.A., Longacre, A., Hsiao, K., Fan, H., Meng, F., Mitchell, J.E., Rauch, J., Ucker, D.S., Levine, J.S., 2006. Apoptotic cells, at all stages of the death process, trigger characteristic signaling events that are divergent from and dominant over those triggered by necrotic cells: Implications for the delayed clearance model of autoimmunity. J Biol Chem 281, 4663-4670.

Rawicz, W., Olbrich, K., McIntosh, T., Needham, D., Evans, E., 2000. Effect of chain length and unsaturation on elasticity of lipid bilayers. Biophysical journal 79, 328-339. Rimm, D.L., Koslov, E.R., Kebriaei, P., Cianci, C.D., Morrow, J.S., 1995. Alpha(1)(E)Catenin Is an Actin-Binding and Actin-Bundling Protein Mediating the Attachment of F-Actin to the Membrane Adhesion Complex. P Natl Acad Sci USA 92, 8813-8817.

Sachs, F., 2010. Stretch-activated ion channels: what are they? Physiology (Bethesda) 25, 50-56.

Sackin, H., 1995. Stretch-activated ion channels. Kidney Int 48, 1134-1147.

Schaefer, A.W., Schoonderwoert, V.T., Ji, L., Mederios, N., Danuser, G., Forscher, P., 2008. Coordination of actin filament and microtubule dynamics during neurite outgrowth. Developmental cell 15, 146-162.

Soedel, W., 2004. Vibrations of shells and plates. CRC Press.

Straight, A.F., Cheung, A., Limouze, J., Chen, I., Westwood, N.J., Sellers, J.R., Mitchison, T.J., 2003. Dissecting temporal and spatial control of cytokinesis with a myosin II inhibitor. Science 299, 1743-1747.

Tsuchiya, S., Yamabe, M., Yamaguchi, Y., Kobayashi, Y., Konno, T., Tada, K., 1980. Establishment and characterization of a human acute monocytic leukemia cell line (THP-1). Int J Cancer 26, 171-176.

Wang, B.R., Bienvenu, C., Mendez-Garza, J., Lancon, P., Madeira, A., Vierling, P., Di Giorgio, C., Bossis, G., 2013. Necrosis of HepG2 cancer cells induced by the vibration of magnetic particles. J Magn Magn Mater 344, 193-201.

Wang, C., Curtis, J.E., Minden, M.D., McCulloch, E.A., 1989. Expression of a retinoic acid receptor gene in myeloid leukemia cells. Leukemia 3, 264-269.

Weliky, M., Oster, G., 1990. The mechanical basis of cell rearrangement. I. Epithelial morphogenesis during Fundulus epiboly. Development 109, 373-386.

Yong, A.S.M., Szydlo, R.M., Goldman, J.M., Apperley, J.F., Melo, J.V., 2006. Molecular profiling of CD34(+) cells identifies low expression of CD7, along with high expression of proteinase 3 or elastase, as predictors of longer survival in patients with CML. Blood 107, 205-212.

Zanini, D., Gopfert, M.C., 2013. Mechanosensation: tethered ion channels. Curr Biol 23, R349-351.

Zhou, Z., Hui, T., Tang, B., Ngan, A., 2014a. Accurate measurement of stiffness of leukemia cells and leukocytes using an optical trap by a rate-jump method. RSC Advances 4, 8453-8460.

Zhou, Z., Sun, X., Ma, J., Man, C., Wong, A., Leung, A., Ngan, A., 2016. Mechanical oscillations enhance gene delivery into suspended cells. Scientific reports 6 .

Zhou, Z.L., Hui, T.H., Tang, B., Ngan, A.H.W., 2014b. Accurate measurement of stiffness of leukemia cells and leukocytes using an optical trap by a rate-jump method. Rsc Adv 4, 8453-8460. 
Zhou, Z.L., Tang, B., Ngan, A.H.W., 2012. The biomechanics of drug-treated leukemia cells investigated using optical tweezers. Nano Life 2, 1-10. 


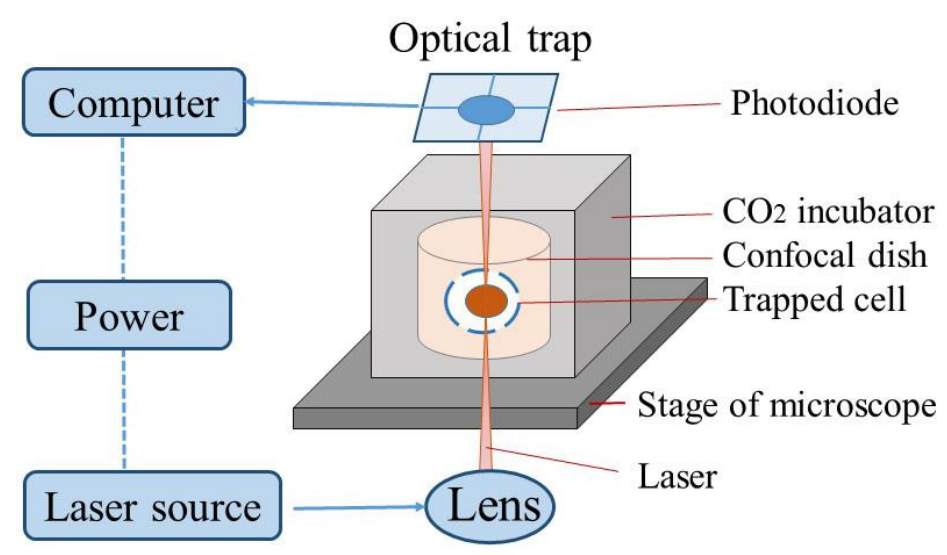

(A)

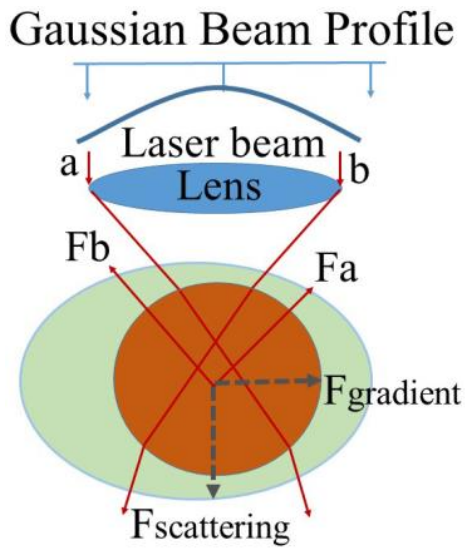

(B)

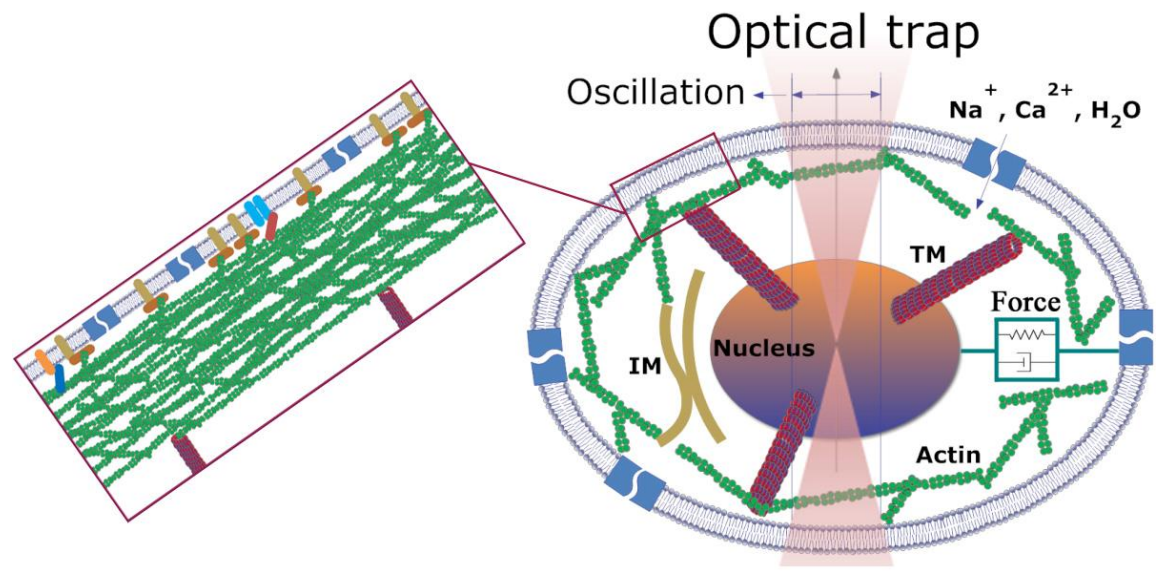

(C)

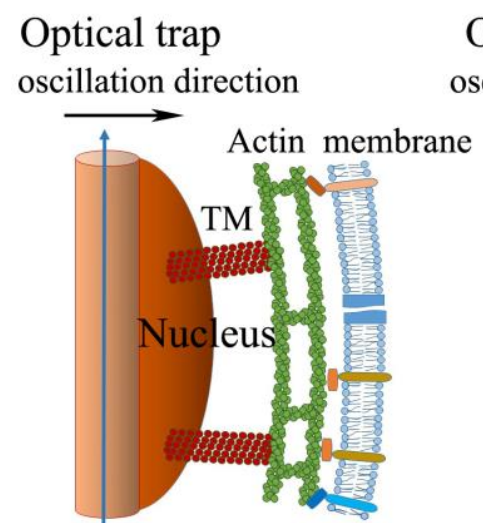

Before
Optical trap oscillation direction

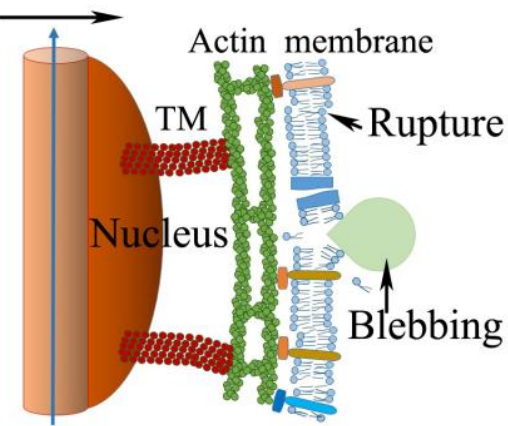

After

(D)

Figure 1. (A) Schematic showing the setup used for cell oscillation by optical trap. (B) Schematic showing the mechanism of a cell nucleus being trapped by a laser beam. (C) Schematic showing nuclear oscillation by optical trap. The oscillatory motion of the trapped nucleus is resisted by the cytoskeleton network comprising tubulin microfilaments (TM), intermediate microfilaments (IM) and actin microfilaments, which in turn transmit forces to upset the cell membrane. (D) Schematic showing a cell oscillated by a laser trap undergoing membrane rupture and blebbing. 


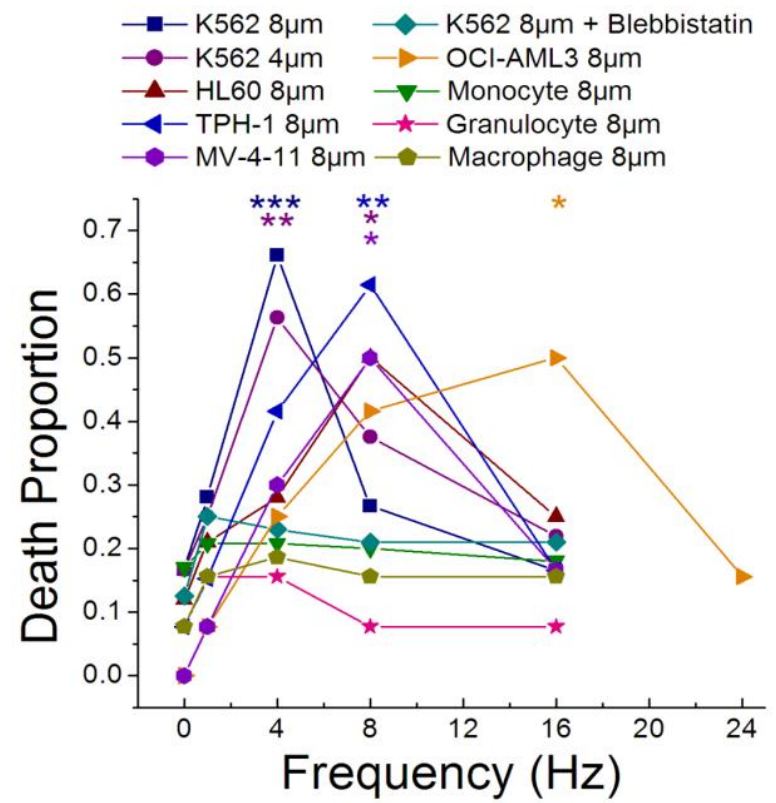

(A)

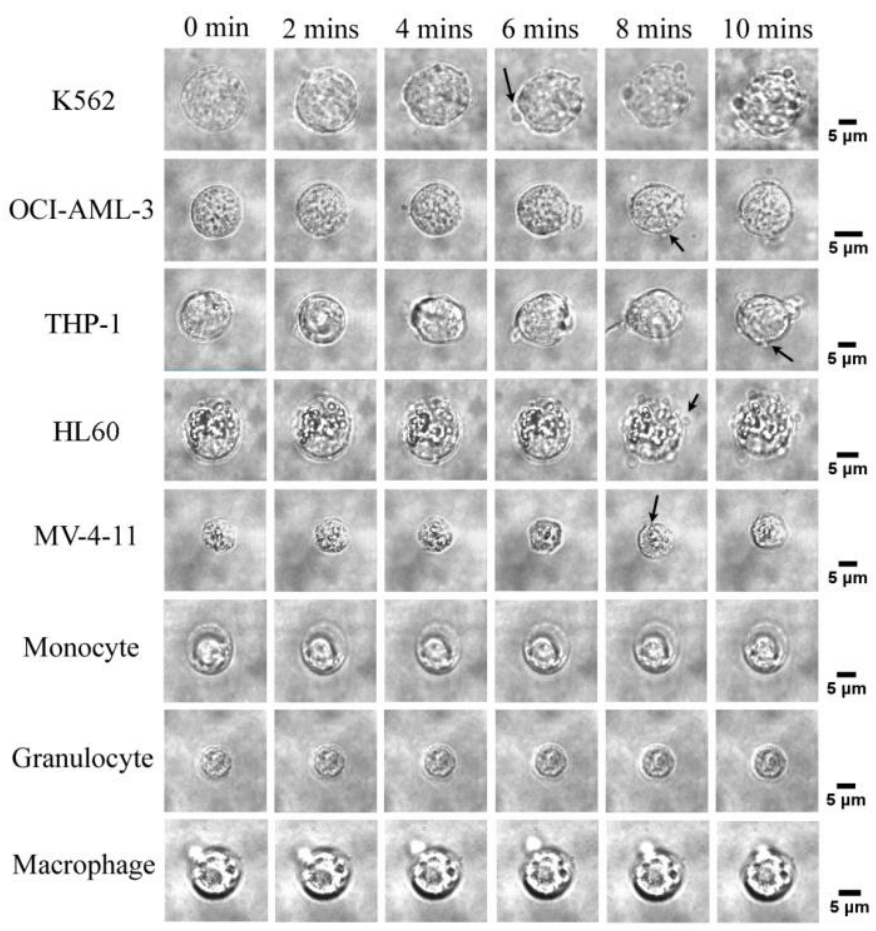

(B)

Figure 2 (A): Death proportion of different types of cells studied versus scanning frequency at scanning amplitudes of 4 or $8 \mu \mathrm{m}$. The experiments were repeated three times for K562 cells, and twice for other cell types. Every time at each frequencyamplitude condition, 8 cells of the same cell type were measured. In total, at least 32 data points were collected at each frequency-amplitude condition for K562 cells, and 24 data points at each condition for other types of cells. Cell membrane blebbing observed under optical light during laser oscillation is considered as an indication of cell death. Stars indicate the $p$ value in the Chi-squared test: $*:<0.05,{ }^{*}:<0.01$ and $* * *:<0.001$. (B): Optical images of different cell types at different time points during the 10-minute oscillations. The oscillation frequency for the K562 and OCI-AML-3 
cells was 4 and $16 \mathrm{~Hz}$, respectively, and that for other types of cells was $8 \mathrm{~Hz}$. The laser-beam amplitude was $8 \mu \mathrm{m}$ in all cases. Membrane blebbing (black arrow pointed) and cell distortions, both indicating cell death, were observed in the leukemia cells.

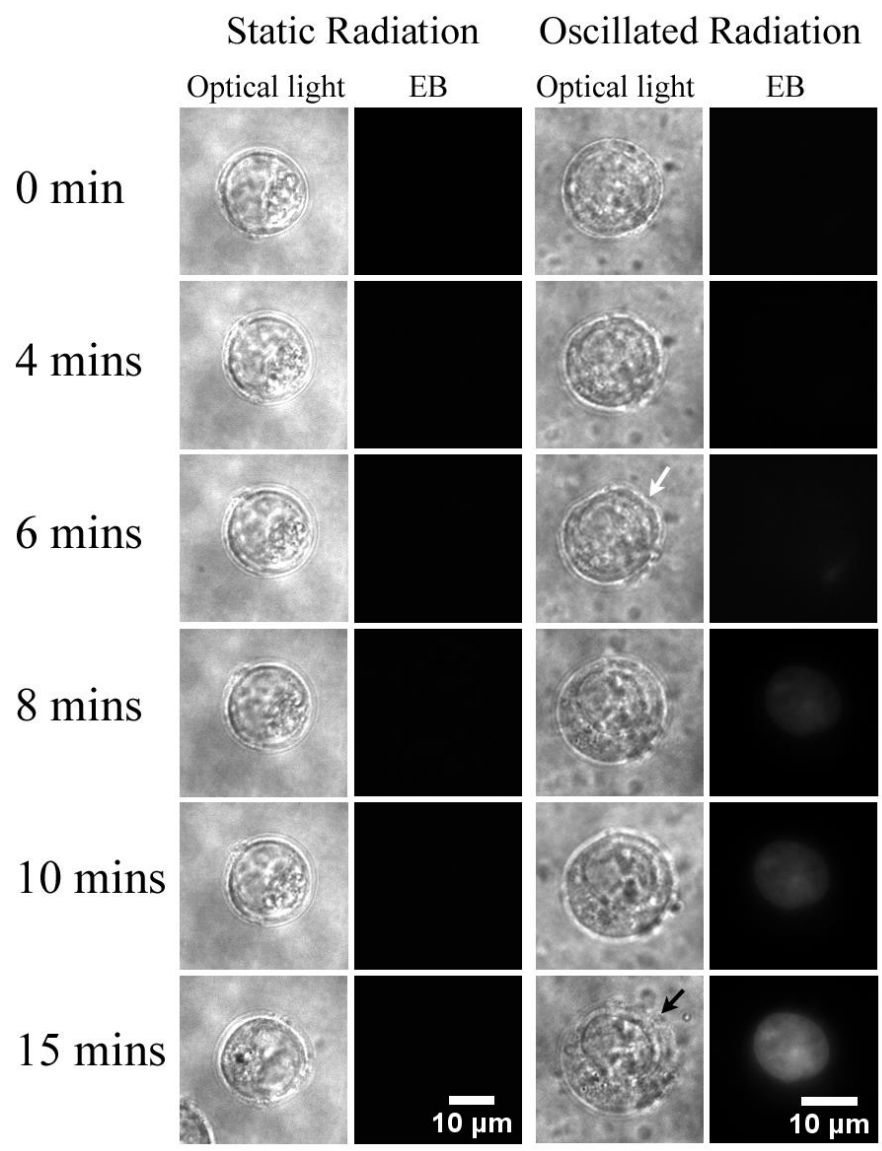

(A) 


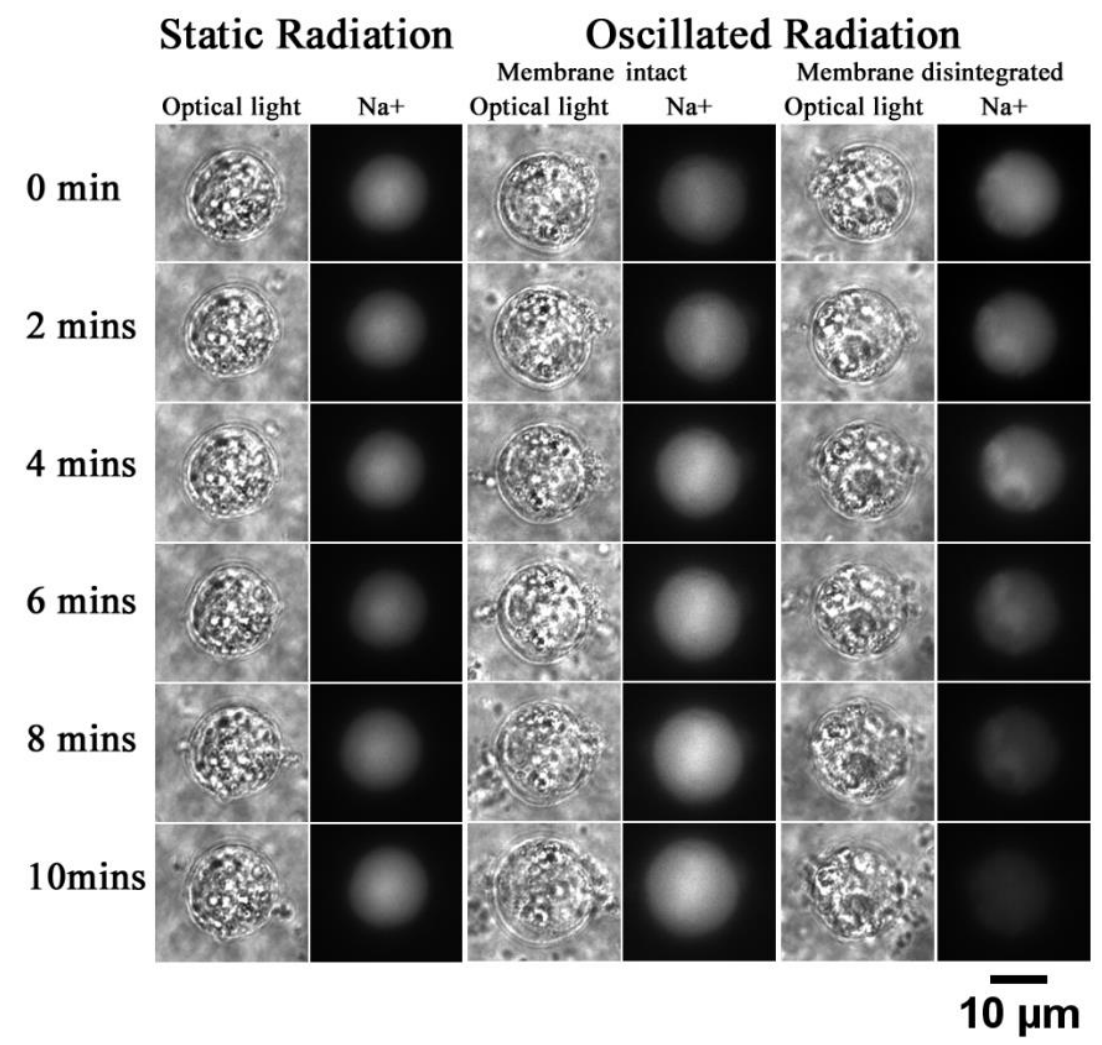

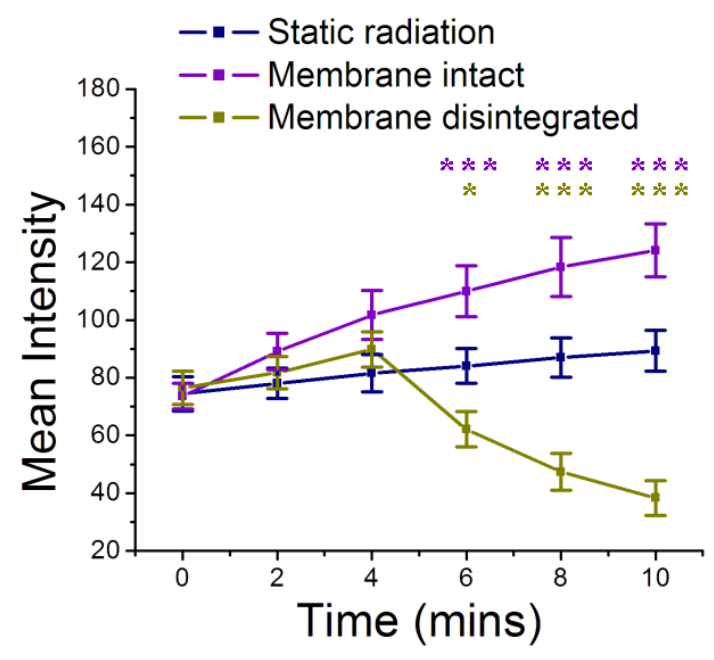

(C)
(B)

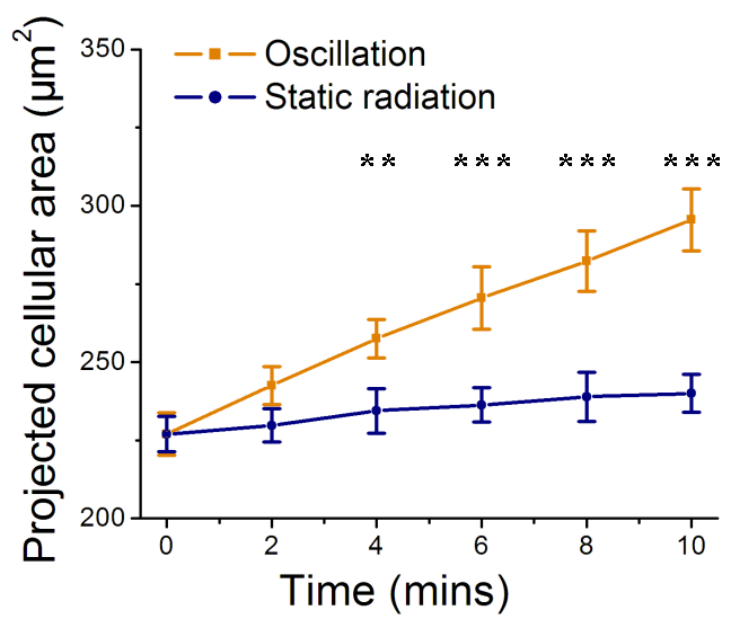

(D)

Figure 3: (A): Optical and ethidium bromide (EB) fluorescence micrographs of K562 cells under static laser irradiation and oscillatory laser irradiation at $4 \mathrm{~Hz}$ and $8 \mu \mathrm{m}$ amplitude for 10 minutes. Cell distortions (white arrow pointed) was observed after 6 minutes of laser radiation, and after 8 minutes of laser radiation positive EB staining was noted, and this increased after the extra 5 minutes of off-laser observation, together with cell volume increase (black arrow pointed); The experiment for the EB staining was repeated 3 times. (B) Micrographs showing $\mathrm{Na}^{+}$ion intensity inside typical K562 cells exposed to the static radiation and radiation with frequency of $4 \mathrm{~Hz}$ and amplitude of $8 \mu \mathrm{m}$ for different time durations. (C) The corresponding mean intensity vs time plots. Scale bars indicate one standard deviation. The experiment for the $\mathrm{Na}^{+}$ion intensity measurement was repeated once. A total of 6 samples was measured in each group at 
each time point, expect the group with disintegrated membrane, for which the sample number is 2 . A significant drop of $\mathrm{Na}^{+}$intensity indicates $\mathrm{Na}+$ leaking from cytoplasm into culture medium and hence membrane rupture. Stars indicate the $p$ values in the two-population $t$ tests: $*:<0.05, * *:<0.01$ and $* * *:<0.001$. The color of the stars indicates the unpaired $t$ tests between the corresponding groups (membrane intact and membrane disintegrated) and the static radiation group. (D) Increase in projected cellular area K562 cells vs laser irradiation time under oscillation frequency of $4 \mathrm{~Hz}$ and amplitude of $8 \mu \mathrm{m}$. All the images were captured at a fixed focus level to make the measured projected areas from different images comparable. The initial cells had diameters of around $17 \mu \mathrm{m}$. Scale bars indicate one standard deviation. A total of six samples was measured in each group at each time point, except that two samples were measured in the membrane disintegrated group in Figure 3(C), for which the $\mathrm{Na}^{+}$ intensity may decrease any time in different measured cells due to alteration of membrane permeability during laser oscillations. Stars indicate the $p$ values in the twopopulation $t$ tests: $*:<0.05$, **:<0.01 and $* * *:<0.001$.

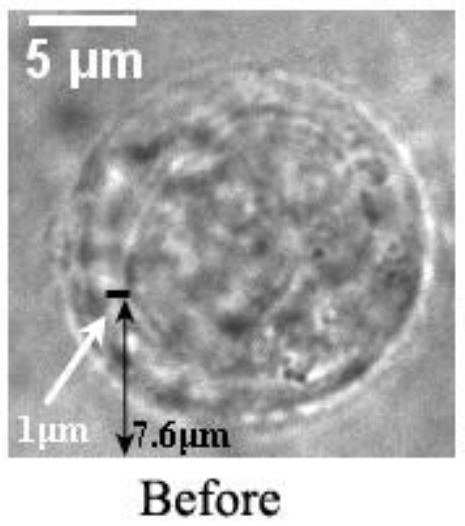

(A)

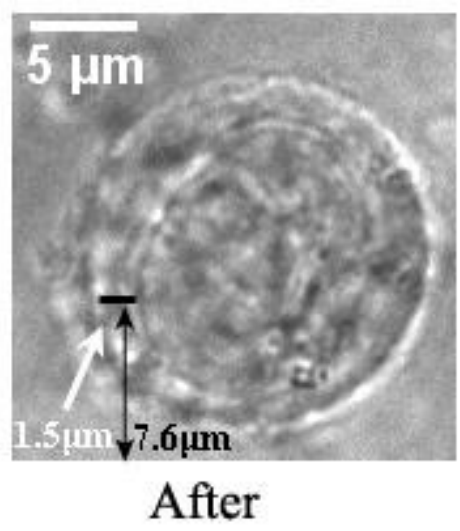

After

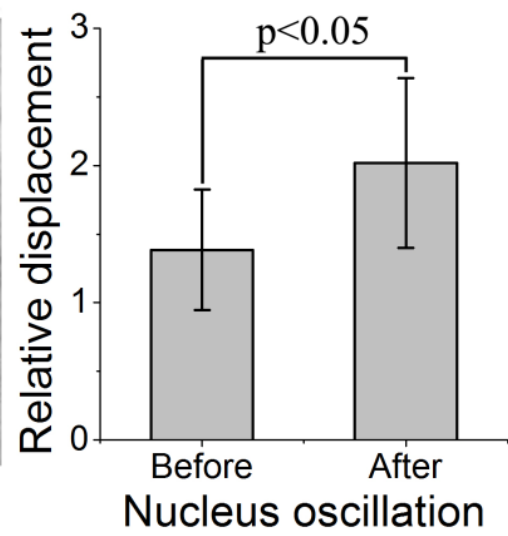

(B)

Figure 4(A) Optical micrographs of a K562 cell before and after laser oscillation at 1 $\mathrm{Hz}$ frequency and $8 \mu \mathrm{m}$ amplitude. The dark lines pointed by the white arrows in both images indicate the position change of the nucleus inside cell in the laser oscillation process. (B) Histogram graph of the relative displacement between cell nucleus and membrane of the K562 cells. $10 \mathrm{~K} 562$ cells collected from 4 independent experiments were measured by using ImageJ (1.46r version). Two-population $t$ test was performed to the experiment, yielding $p<0.05$ which indicates that the displacement between cell nucleus and membrane was significantly different after the K562 cells were subjected to laser oscillation. 


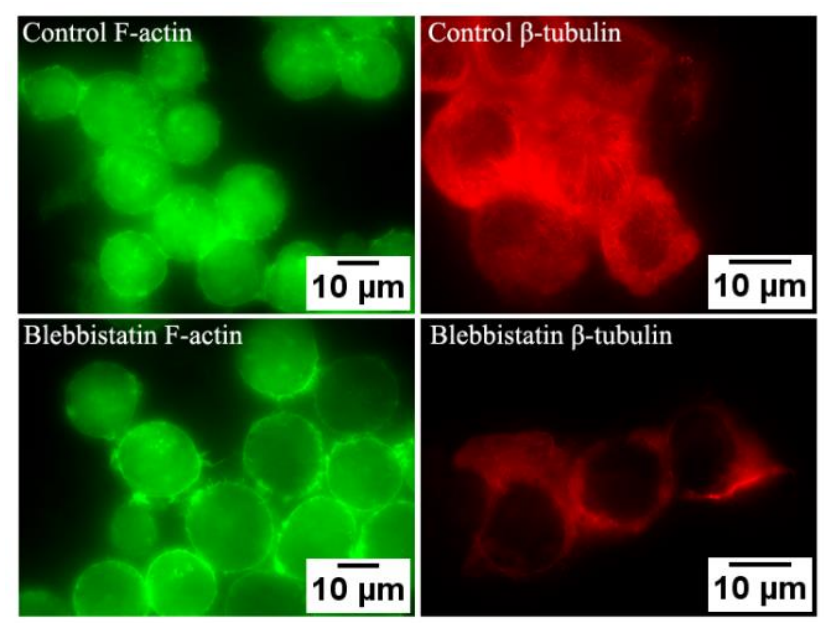

(A)

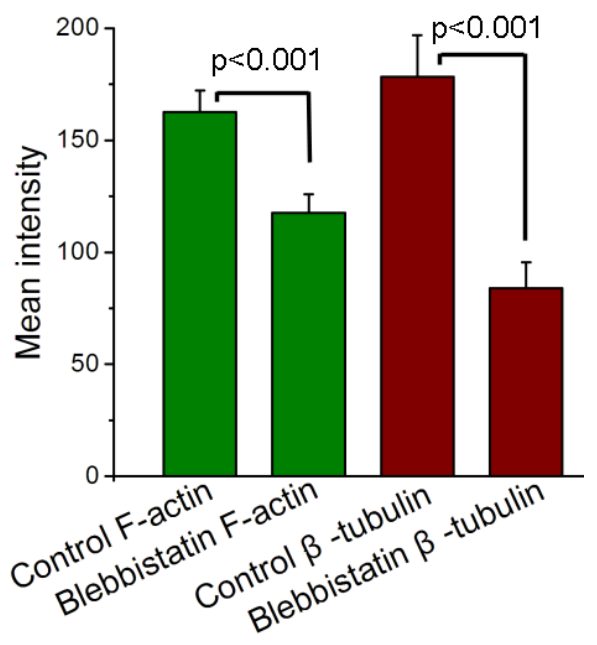

(B)

Figure 5: (A) Fluorescence micrographs showing F-actin and $\beta$-Tubulin filaments of K562 cells with and without Blebbistatin treatment; (B) Mean fluorescent intensities of the F-actin and $\beta$-Tubulin filaments stained by phalloidin-FITC and anti- $\beta$-tubulin-Cy3, of K562 cells with and without Blebbistatin treatment. The experiment was repeated once. A total of 78 cells in each group was measured to determine their filament intensities using ImageJ (1.46r version). Scale bars represent one standard deviation. Two-population $t$-tests indicate that K562 cells without Blebbistatin treatment exhibit significantly denser F-actin and $\beta$-Tubulin filaments than cells with Blebbistatin treatment $(p<0.001)$. 


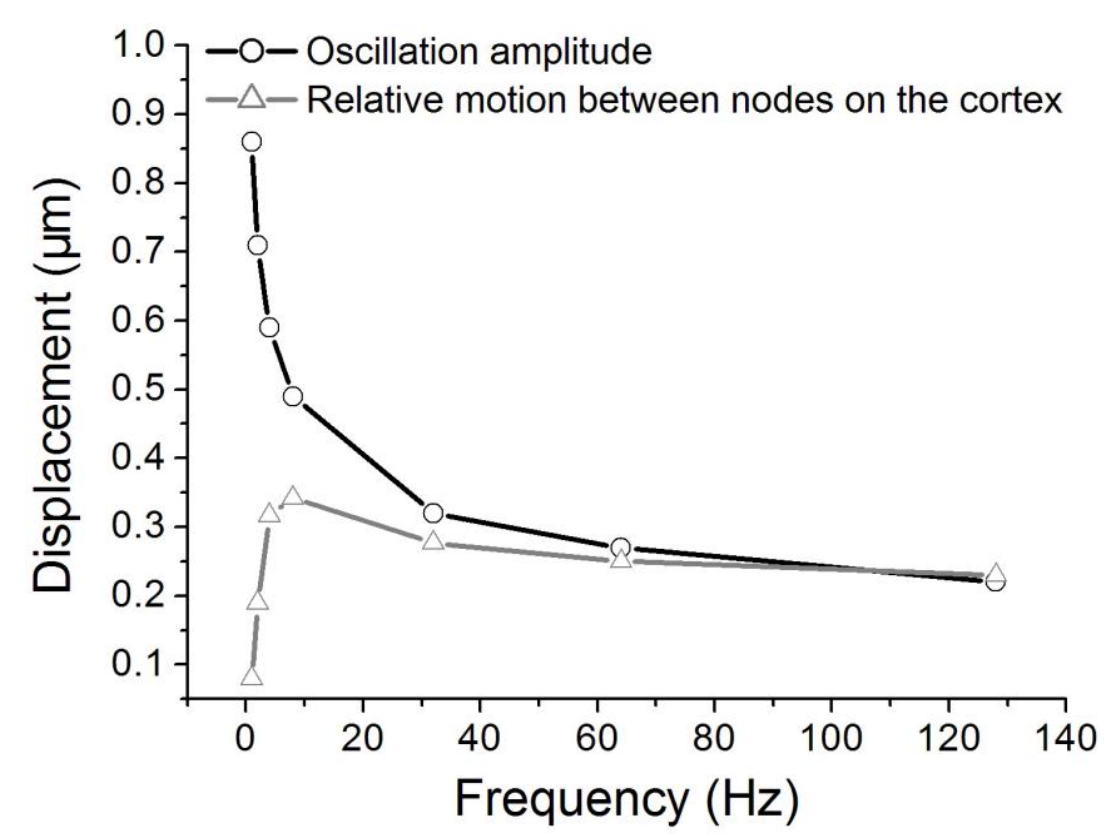

(A)

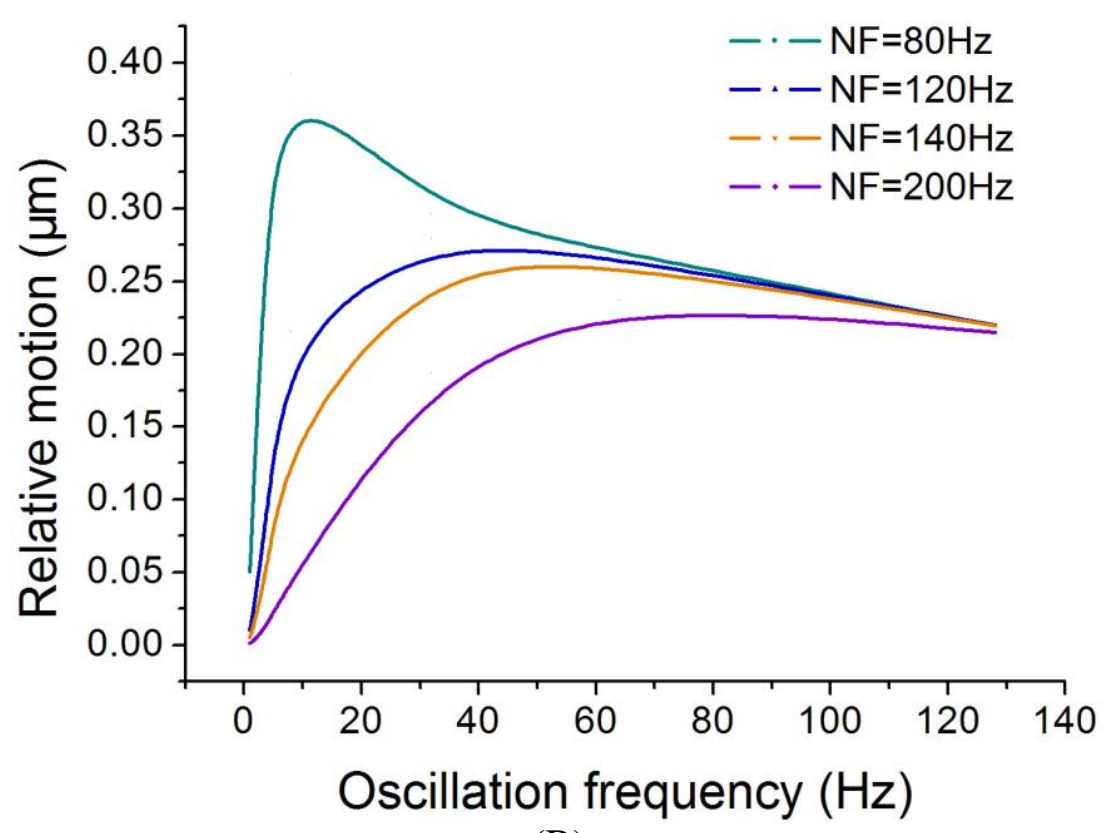

(B)

Figure 6. (A) Modelled oscillations of cytoskeleton cortex. Plots of the average relative motion between actuation nodes and the driven nodes, and oscillation amplitude of nucleus vs. frequency. The average relative motion is the difference between the displacements of the driving nodes at 0.5 cycle and the root-mean-square of the nodal displacements at 0.5 cycle. The relative motion can indicate the stretching of the cortex. Videos showing the cortex simulations are provided as Supplementary Materials. (B) Relative motion between an actuation node and a typical driven node vs oscillation frequency in cytoskeleton cortex structures of different natural frequencies (NF), calculated according to eqn. (2) at $\omega t=\frac{\pi}{2}$ when the distortion is most serious. Model parameters are given in Supplementary Material (I, II). 
Table - Cell types investigated

\begin{tabular}{|c|c|c|}
\hline Cell type & Leukemia type and control & Reference \\
\hline K562 & Chronic myelogenous leukemia & $\begin{array}{l}\text { (Lozzio and } \\
\text { Lozzio, 1975) }\end{array}$ \\
\hline OCI-AML-3 & $\begin{array}{l}\text { Acute myelogenous leukemia } \\
\text { (AML FAB M4) }\end{array}$ & (Wang et al., 1989) \\
\hline THP-1 & Acute monocytic leukemia & $\begin{array}{c}\text { (Tsuchiya et al., } \\
\text { 1980) }\end{array}$ \\
\hline HL60 & $\begin{array}{c}\text { Acute promyelocytic leukemia, } \\
\text { mainly a type of neutrophilic } \\
\text { promyelocytes }\end{array}$ & $\begin{array}{c}\text { (Gallagher et al., } \\
\text { 1979) }\end{array}$ \\
\hline MV-4-11 & $\begin{array}{c}\text { Acute monocytic leukemia } \\
\text { leukemia carrying } \mathrm{t}(4.11) \\
\text { translocation }\end{array}$ & (Lange et al., 1987) \\
\hline Monocyte & $\begin{array}{l}\text { Normal white blood cells, as } \\
\text { control }\end{array}$ & \\
\hline Granulocyte & ditto & \\
\hline Macrophage & ditto & \\
\hline
\end{tabular}

